\title{
Hip Joint Laxity in Small Dog Breeds A Radiological Study
}

\author{
Jens Arnbjerg*
}

Department for Diagnostic Imaging, Faculty of Health and Medical Sciences, University of Copenhagen, Dyrlaegevej 32, DK 1870 Frederiksberg C,

Received: 27 December, 2016; Accepted: 8 March, 2017; Published: 17 March, 2017

*Corresponding author: Jens Arnbjerg, Department for Diagnostic Imaging, Faculty of Health and Medical Sciences, University of Copenhagen, Dyrlaegevej 32, DK 1870 Frederiksberg C, Denmark, Tel:+45 43967126; Fax:+45 35282929; E-mail: jarn@image.dk

\begin{abstract}
The clinical and hereditary significance of laxity of the hip joints in dog breeds of small size is not earlier described. Joint laxity of the hip joints was measured in 55 dogs recently euthanised for other reasons than orthopaedic or back problems by distally drawing in the hocks with up to $20 \mathrm{~kg}$ to provoke laxity in the hip joints. Stress radiographs of the hips in the extended position were then taken at 0,12 and $20 \mathrm{~kg}$ traction. Hip joints with any signs of osteoarthrosis on the radiographs were excluded from the study. The displacement index, I, was calculated as the displacement of the caput femoris from the cranial border of the acetabulum (d) divided by the radius of the caput femoris (r). The greatest laxity was found in the Dachshund having a mean I of 1.27 and the least laxity was found in Breton Basset dogs having a mean I of 0.25 . There was no statistically significant in age, weight or sex related variation in laxity and little individual difference in displacement within the breeds but highly statistically significant effect of breeds $(\mathrm{p}<0.0001)$ on I.
\end{abstract}

Vacuum phenomenon was seen in distracted hips with high I.

The study indicates that joint laxity can be present without clinical importance and not predispose for osteoarthritis in dog breeds of small size.

Key words: Hip Subluxation small Dog Breeds; Subluxation and HD; Tension on Joints; Hip Distraction; Vacuum Phenomenon

\section{Introduction}

It is believed that a major reason in large breeds of dogs $(20-35 \mathrm{~kg}$ ) for development of Hip Dysplasia with secondary osteoarthritis is abnormal joint laxity [1-3].

For smaller dog breeds, HD-prevalence's have been reported very low: Scottish terrier $0.12 \%$ Miniature Schnauzer $1.5 \%$ and $3.2 \%$ for the Dachshund $[4,5]$.

However, in some small dog breeds, cranio-caudal laxity can be found without causing any joint deformity or osteoarthritis even at an old age and this leads to some considerations why they do not develop osteoarthritis [6].

Having experienced patients with hyper mobility of the hipand shoulder joints without showing any clinical signs, it was decided to look at the degree of distal laxity present in hip joints without osteoarthritis by stressing the joint with an exact amount of weight (12 and $20 \mathrm{~kg}$ ) in dog breeds of small size.

\section{Material \& Methods}

All dogs were euthanized due to other reasons than back and/or hind leg problems at the University Hospital for Small Animal with an overdose of barbiturate less than 20 minutes before the stress procedure were performed. All 55 dogs of 15 different breeds having a body weight of $16 \mathrm{~kg}$ or less and age from 3 to 14 years had their Coxo-Femoral joints radiographed in ventrodorsal projection in dorsal recumbence position (Siemens Polydores LX50, Fuci Film and 3M Trimax intensifying screens).

The chest was fixed and tension was applied equally to each leg with a chain around the hocks connected to spring-loaded weight via a bar across both hocks.

The dogs were radiographed, first with no tension on the hips figure 1 and then with tension (measured with the spring balanced weight) on both hind legs of $12 \mathrm{~kg}$ figure 2 and $20 \mathrm{~kg}$

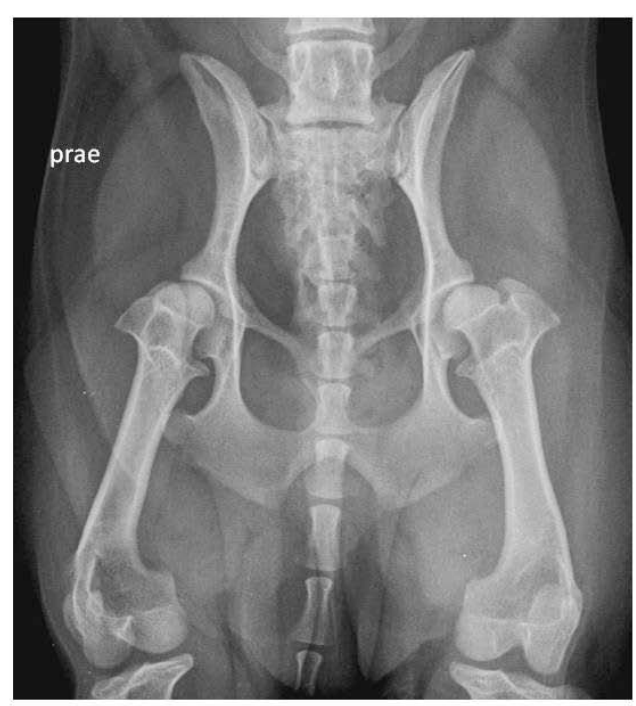

Figure 1: Radiograph showing the Pelvis taken in VD projection of a Dachshund in dorsal recumbency taken before tension is put on the hind legs $(0 \mathrm{~kg})$. 
figure 3 and finally after the stressing with no tension applied on the hips (Figure 4). As the manually applied traction in the hip joints is not measurable during clinical procedure of taking radiographs of the hip the described procedure was chosen as an accurate method for measuring the stress by blocking the weight during the radiographic exposure.

None of the dogs were euthanized due to clinical orthopaedic reasons. Two dogs showing osteoarthritis on the first radiographs were excluded from the study. All the euthanized dogs hips were macroscopically examined by removing the muscles around

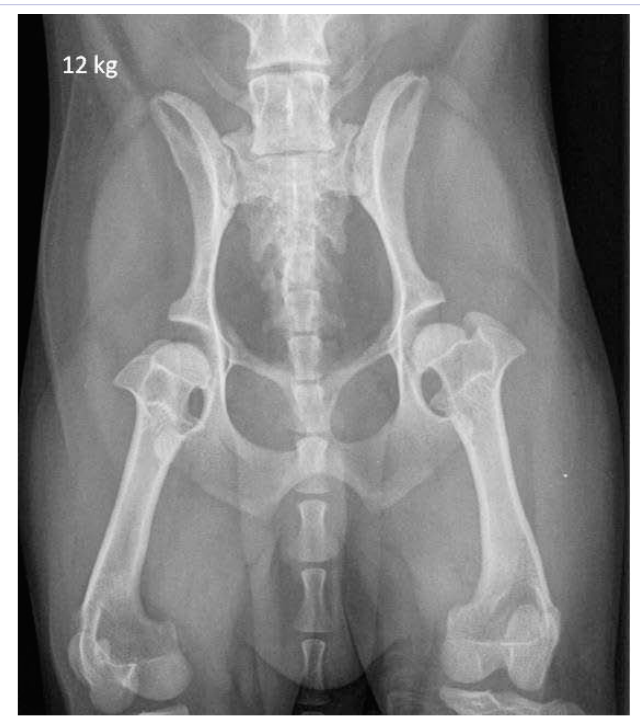

Figure 2: Radiograph of the same Dachshund and same position as figure 1 taken during $12 \mathrm{~kg}$ of tension in distal direction (12 kg). Notice there is significant dislocation of the femoral heads in distal direction.

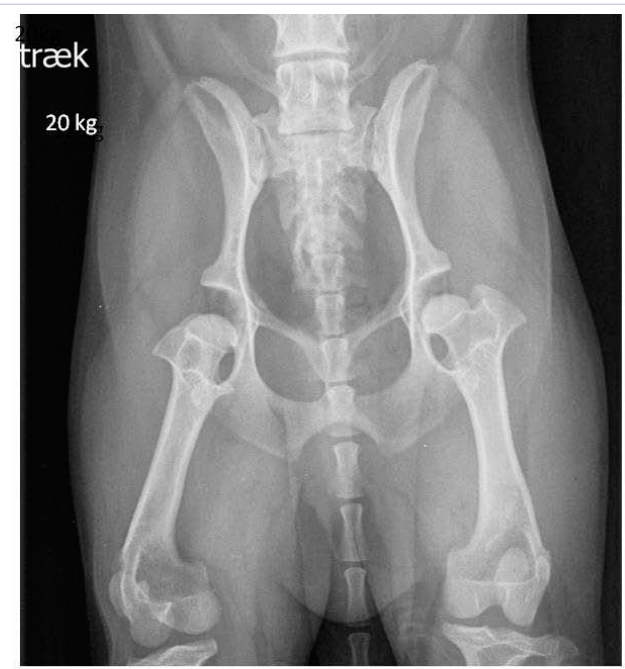

Figure 3: Radiograph of the same Dachshund and same position as figure 1 taken during $20 \mathrm{~kg}$ of tension in distal direction $(20 \mathrm{~kg})$. Notice there is a further dislocation of the femoral heads in distal direction compared with figure 2 (12 kg tension).

Displacement Index at $20 \mathrm{~kg}$ is 1.30 for this dog.

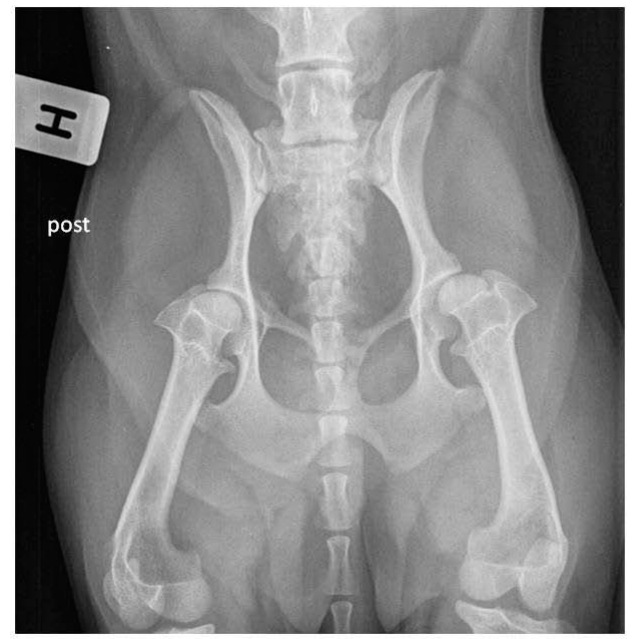

Figure 4: Radiograph showing the same Dachshund and same position as figure 1. taken after release of tension in distal direction $(0 \mathrm{~kg}$ p) Notice there is no dislocation of the femoral heads in distal direction and the picture is very similar to figure 1 .

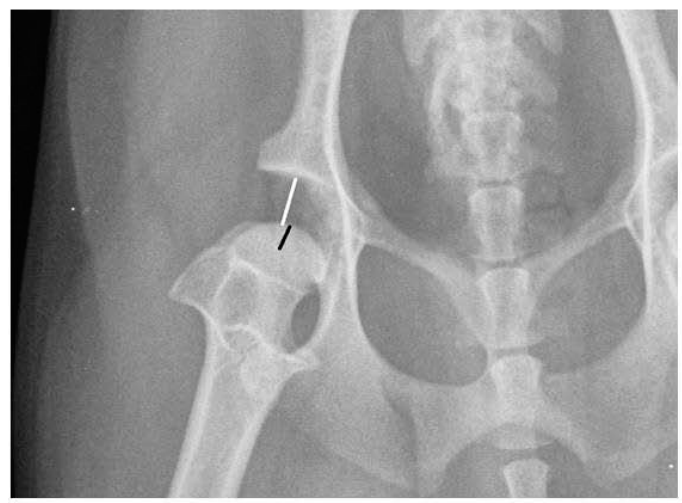

Figure 5: Radiograph showing the method of measuring radius of caput Femuris (r) (black line) and the dislocation (d) (white line).

the hip and the hip joints were opened after the radiographic procedures.

2 of the dachshunds and 2 of the Spaniels and 2 Fox Terrier were radiographed with a piece of wood $(1.5 \mathrm{~cm} \times 4 \mathrm{~cm} \times 15 \mathrm{~cm})$ to observe the laxity in abaxial plan (modified Penn Hip method as the normal bar does not fit in these small dogs).

The laxity was measured on the radiographs taking during the distal traction by two trained persons and the distal Displacement Index was calculated measuring the radius ( $r$ ) of the femoral head and the caudal displacement of the femoral head from the cranial contour of Acetabulum (d). Displacement index, $\boldsymbol{I}$, were calculated $(\mathrm{d} / \mathrm{r})$ figure 5 as the displacement of the Caput Femoris from the cranial border of the acetabulum (d) divided by the radius of the Caput Femoris (r) on the radiographs taken at $20 \mathrm{~kg}$ tension ( $10 \mathrm{~kg}$ on each leg) compared with 0 tension (Table $1,2)$. Both joints were examined and the index calculated. The mean value for each dog were used in the statistical calculation. 
The statistical calculation was made by the SAS procedure GLM (Generalized Linear Model).

After taking the radiographs the hip joints were examined macroscopically.

\section{Results}

No secondary arthrotic changes were observed in the hip joints in this material and all hip-joints were considered radiographically and macroscopically normal for the specific breed.

None of the dogs had ruptured the caput femoris ligament or showed macroscopically any cartilage/synovial membrane changes, when inspected after the measurements.

There were no sex, body-weight or age related

significant difference in the displacement of caput femoris within any of the individual breeds (Table 1 and Figure 4). The age of all dogs was more than 3 years and the average for all 55 dogs were 7.4 years. There was no statistically significant age, weight or sex related variation in laxity and little individual difference in displacement within the breeds giving a highly statistically significant effect of breeds ( $p<0,0001)$. Vacuum phenomenon

Table 1a: An overlook over 55 dogs with the breeds arranged according to high (A) and low (B) Displacement-Index. The Dachshund showing the highest and Breton Basset and Fox terrier showing the lowest displacement-Index. Notice the age is more than 3 years for all dogs. A. High laxity: I $>0.50$

\begin{tabular}{|c|c|c|c|c|c|}
\hline Breed & Male & Female & $\begin{array}{c}\text { Weight } \\
\text { Kg }\end{array}$ & $\begin{array}{c}\text { Age } \\
\text { Years }\end{array}$ & $\begin{array}{c}\text { Index at } \\
\mathbf{2 0 ~ k g} \\
\text { tension }\end{array}$ \\
\hline Boston Terrier & 1 & 1 & 13 & 10 & $0.57-0.66$ \\
\hline Cairn Terrier & 1 & 1 & $9-10$ & $8-10$ & $0.66-0.72$ \\
\hline Dachshund & 6 & 9 & $6-13$ & $6-13$ & $1.18-1.33$ \\
\hline Pekingese & 1 & 1 & 6 & 12 & $0.98-1.21$ \\
\hline Pincher Mini & 1 & 0 & 6 & 4 & 0.83 \\
\hline Poodle Mini & 0 & 2 & 6 & $12-13$ & $0.95-1.19$ \\
\hline Sealyham Ter. & 2 & 1 & 6 & 3 & $0.67-0.72$ \\
\hline Yorkshire Ter. & 1 & 1 & 7 & 6 & $0.50-0.58$ \\
\hline
\end{tabular}

\section{Table 1b:}

B. Low laxity: $\mathrm{I}<0.40$

\begin{tabular}{|c|c|c|c|c|c|}
\hline Breed & Male & Female & $\begin{array}{c}\text { Weight } \\
\text { Kg }\end{array}$ & $\begin{array}{c}\text { Age } \\
\text { Years }\end{array}$ & $\begin{array}{c}\text { Index at } \\
\text { 20 kg tension }\end{array}$ \\
\hline $\begin{array}{c}\text { Breton } \\
\text { Basset }\end{array}$ & 1 & 1 & 16 & 5 & 0.25 \\
\hline $\begin{array}{c}\text { Bulldog } \\
\text { French }\end{array}$ & 1 & 1 & $14-15$ & 3 & $0.25-0.28$ \\
\hline Fox Terrier & 4 & 3 & $7-10$ & $6-13$ & $0.25-0.36$ \\
\hline Maltese & 2 & 1 & $4-5$ & 9 & $0.25-0.30$ \\
\hline Papillion & 1 & 1 & $5-6$ & $3-4$ & 0.33 \\
\hline Spaniels & 3 & 5 & $6-11$ & $6-13$ & $0.33-0.40$ \\
\hline $\begin{array}{c}\text { WHW } \\
\text { terrier }\end{array}$ & 2 & 0 & 7 & $9-10$ & 0.40 \\
\hline
\end{tabular}

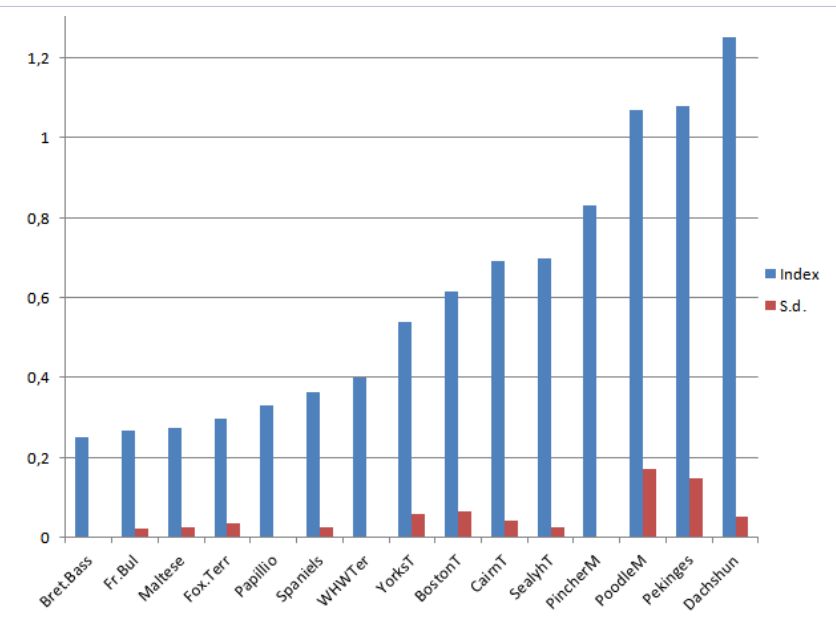

Table 2: Showing the different breeds arranged in accordance to the specific Index (blue column) in the breeds at the tension of $20 \mathrm{~kg}$. The Maltese and Fox Terrier having lower I-values The Spaniels, Boston Terrier and Cairn Terrier showing medium and Mini Poodle, Pekingese and Dachshund showing high $\boldsymbol{I}$-values.

The standard deviation (s.d.) is low for all breeds (red column).

was seen in distracted hips with high I.

The dogs showed that an increasing tension resulted in a breed related difference in a gradually caudal dislocation of the femoral head and the dislocation was proportional to the tension up to $20 \mathrm{~kg}$ (Figure 2,3).

There was little within-breed difference between the dogs giving a highly statistically significant difference between breeds. The Dachshund had the highest (I: 1.27), Mini Poodle, Pekingese, Sealyham Terrier, West Highland White Terrier and Cairn Terrier having also a high laxity. The Yorkshire Terrier had results near the middle values; Breton Basset (lowest $I$ : 0.25), French Bulldog, Spaniels, Boston-, Fox Terrier, Maltese and Papillon being more rigid than the middle value at $20 \mathrm{~kg}$ tension (Table 1,2).

In the Pekingese, Dachshund and Cairn Terrier the caput femoris could be drawn $6-12 \mathrm{~mm}$ distally depending on the amount of tension. The radiographs taken after release of tension showed the same or very similar position of the femoral head as before the first tension.

The Table 2 is a graphic visualization of the entire material in relation to the different breeds, where the Dachshund was having the highest Index and Breton, Fr . Bulldog, Maltese and Fox-Terrier having the lowest Index. The standard deviation is generally low.

As shown in Figure 3 there were often seen a black area between caput femoris and acetabulum (Vacuum Phenomenon) at 12 and $20 \mathrm{~kg}$ tension in those dogs hawing a high Displacement Index.

At macroscopic autopsy none of the dogs showed any evident sign of abnormality around the pelvic joints including 
hemorrhage, changed diameter or ruptured caput femoris ligament, but the dogs with high laxity were easier to move, than those in the non-laxity group - especially after destroying the hip joint capsule.

The Dachshund showed some laxity at the modified Penn Hip method, whereas the Fox Terrier and Spaniels could not be dislocated, but due to inconsistency in reproducing results, this method was only performed on 6 dogs.

\section{Discussion}

When radiographing dogs under mild sedation and even under anaesthesia some tension will always be put on the hind legs during the radiographic procedure. In this study an exact amount of tension results in some breeds in a displacement of caput femoris away from its normal position in the acetabulum (Figure 2,3). In this study the drawing of the femoral head was in a caudo-distal direction and therefore moving the femoral head in a distal direction. This could explains why the femoral head can move without destroying the ligament, but it does not explain why there is laxity in some breeds and not in others as a result of exactly the same tension $-20 \mathrm{~kg}$. The amount of tension was similar to the tension put on human legs in the study of Arvidsson in relation to the difference in bodyweight [7].

There were no significant age related, sex and weight variations in the laxity $\boldsymbol{I}$ within the breeds which is shown in table 1,2, where it is seen that the Dachshund shows the highest $(1.18$ - 1.33) and Breton Basset and Fox terrier showing the lowest displacement- Index (0.25). Therefore the presence of joint caudal laxity in the hip joints has probably been present to a similar extend constant throughout life. As the age of all dogs is more than 3 years and the average is 7.4 years the secondary osteoarthritis should have developed at this age, if the laxity was causing any trauma to the joint. Due to technical problems, the iron chain did not put exactly the same direction-tension to boths legs in all cases, and a slightly changed rotation was observed in a few cases. The same reason is accepted in case of lack of parallel position of femurs. These findings are in agreement with the fact that prevalence for osteoarthritis in small breed dogs being very low [5]. Therefore the laxity does not seem to result in osteoarthritis in these small breed dogs.

Taken into consideration that as little drawing as only 6 $\mathrm{kg}$ in one leg can produce subluxation in the hip joints in some small dogs might be unreliable or over-diagnosed - especially in young and/or multi-traumatic patients where the pelvic area is involved. These problems are magnified if the position of the dogs is not optimal e.g. in relation to evaluation of patients after injuries where pain often makes it difficult to get the correct position without applying great tension on the leg.

Even it is very difficult to put abaxial pressure on the hip joints due to the very short femur it was tried with a special devise (piece of wood - like the PennHip method), it was possible to luxate the femoral head in the Dachshunds - but it was found to be very individual and difficult to reproduce and therefore only a 6 dogs were tested.
In human trials in planned drawing treatment for joint diseases, relaxation of the muscles associated with the joints involved it has been shown that the muscles is the most important factor in the joint laxity in distal movement [7]. Vacuum Phenomenon was also observed in people at a certain amount of drawing like it is observed in this material in dogs with high laxity as seen in figure 3,4 where air is observed between Caput Femoris and Acetabulum [7].

The dogs reaction in the hips in this material are comparable with anesthetized dogs and the reposition after the traction shows that the muscles retracted after the tension as seen in figure 4, where caput Femoris has the same position as in (Figure 1). The fact that no macroscopic damage could be observed after the radiological procedure shows that the laxity is not a result of damage of the joint or muscles involved. This is an indicator that this model is very realistic compared with a live dog model.

The fact that the femoral heads are moving back to the same position as it was before the tension was applied can explain, as it appears to be the case in man with the same joint-laxity by the muscle tension having a major role to play as a protecting effect to maintaining joint stability [7]. The role of the joint capsule and the ligaments for joint stability is unclear both in man and in animals.

This laxity phenomenon has not been shown in dogs before, and these findings show that in this material there are no direct correlation between the joint laxity and joint-diseases, e.g. hip joints osteoarthritis, in these dog breeds of small size. The laxity is very much depending on the specific breed as some breeds have very little laxity response at the same tension. The findings in this study with subluxation of the hips, but no subsequent osteoarthritic changes in breeds with high $\boldsymbol{I}$, are in accordance with Culp et al who found that the Norberg Angle was an inaccurate predictor of degenerative hip joint disease in six out of seven breeds [8].

Vacuum phenomenon can however, be observed in babies without exposing the hip joints to stress [9]. In human the vacuum phenomenon does not seem to be related to any pathological processes or clinical significance at all [10].

When screening for HD in small dog breeds this laxity can result in a high HD score due to subluxation and thereby not allowing dogs to breed on false grounds if the screening is done according to the same parameters in small and large dog breeds, as subluxation does not seem to result in osteoarthritis in some small breed dogs as shown in this material.

\section{Conclusion}

This material shows that in these smaller sized dogs caudal laxity in the hip joint can be seen as a normal finding, when tension on the hips are just a few $\mathrm{kg}$ and marked if the tension is 6 or more kg on the relaxed (sedated/anesthetised) hip joint. The degree of laxity in hips is significantly dependent on the breed. A degree of laxity that might be considered high and in larger dog breeds can be related to developing HD according to the Penn Hip theory can be present without signs of secondary joint disease in 
some small dog breeds and therefore without clinical significance for these breeds [1].

Therefore distal joint laxity in the small dog breeds might be over-diagnosed as a pathological finding, when the radiographs are taken under some traction and/or in an oblique position.

\section{Acknowledgements}

The author wish to thank Assoc. Prof. Knud A. Christensen for his help with the statistical analyses.

\section{Reference}

1. Smith GK, LaFond E, Heyman SJ, Cofone MA, Gregor TP. Biomechanical characterization of passive laxity of the hip joint in dogs. Am J Vet Res. 1997;58(10):1078-1082.

2. LaFond E, Smith GK, Gregor TP, McKelvie PJ, Shofer FS. Synovial fluid cavitation during distraction radiography of the Cox femoral joint in dogs. J Am Vet Med Assoc. 1997; 210(9):1294-1297.

3. Kapatkin AS, Gregor TP, Hearon K, Richardson RW, McKelvie PJ, Fordyce $\mathrm{HH}$, et al. Comparison of two radiographic techniques for evaluation of hip joint laxity in 10 breeds of dogs. J Am Vet Med Assoc. 2004;224(4):542-546.
4. Witsberger TH, Villamil JA, Schultz LG, Hahn AW, Cook JL. Prevalence of and risk factors for hip dysplasia and cranial cruciate ligament deficiency in dogs. J Am Vet Med Assoc. 2008;232(12):1818-1824. doi: 10.2460/javma.232.12.1818

5. Rettenmaier JL, Keller GG, Lattimer JC, Corley EA, Ellersieck MR. Prevalence of canine hip dysplasia in a veterinary teaching hospital population. Vet Radiol Ultrasound. 2002;43(4):313-318.

6. Fuglede S: Prevalence of arthrosis in the Hip joints of small breed dogs. 2010. Diss. Copenhagen University, DK, Faculty of Life Sciences.

7. Arvidsson I. The Hip Joint: Forces needed for Distraction and appearance of the Vacuum Phenomenon. Scand J Rehab Med. 1990;22(3):157-161.

8. Culp WTN, Kapatkin AS, Gregor TP, Powers MY, McKelvie PJ, Smith GK. Evaluation of the Norberg angle threshold: a comparison of Norberg angle and distraction index as measures of coxofemoral degenerative joint disease susceptibility in seven breeds of dogs. Vet Surg. 2006;35(5):453-459.

9. Zhenjiang L, Wei Y, Lijun Z: Analysis of the vacuum phenomenon in plain hip radiographs in children. Int J Clin Exp Med. 2015;8(3):33253331. 